\title{
Plasma RIPK3 And HMGB1 Predict Severe COVID-19 Progression In ICU Patients: A Single-Center Cohort Study
}

\author{
Katharina Ruskowski \\ University Hospital Frankfurt \\ Holger Neb \\ University Hospital Frankfurt \\ Steven R Talbot \\ Hannover Medical School \\ Suma Choorapoikayil \\ University Hospital Frankfurt \\ Elisabeth H Adam \\ University Hospital Frankfurt \\ Andreas von Knethen \\ University Hospital Frankfurt \\ Kai Zacharowski \\ University Hospital Frankfurt \\ Ulrike Heinicke ( $\nabla$ ulrike.heinicke@kgu.de) \\ University Hospital Frankfurt https://orcid.org/0000-0002-0016-8124
}

Research

Keywords: Necroptosis, Cell Death, COVID 19, Severe Acute Respiratory Syndrome, Intensive Care Units

Posted Date: November 19th, 2021

DOI: https://doi.org/10.21203/rs.3.rs-1064345/v1

License: (ㄷ) (i) This work is licensed under a Creative Commons Attribution 4.0 International License. Read Full License

Version of Record: A version of this preprint was published at American Journal of Respiratory Cell and Molecular Biology on April 6th, 2022. See the published version at https://doi.org/10.1165/rcmb.2022-0039LE. 


\section{Abstract}

Background: Severe progression of coronavirus disease 2019 (COVID-19) causes respiratory failure and critical illness. Recently, these pathologies have been associated with necroptosis, a receptor-interacting serine/threonine-protein kinase 3 (RIPK3) dependent regulated form of inflammatory cell death. Investigations of indicator necroptosis proteins like RIPK3, mixed lineage kinase domain-like pseudokinase (MLKL), receptor-interacting serine/threonine-protein kinases 1 (RIPK1), and high-mobility group box 1 (HMGB1) in clinical COVID-19 manifestations are lacking.

Methods: A prospective prolonged cohort study including 46 intensive care unit (ICU) patients classified with moderate and severe COVID-19 was conducted with daily measured plasma levels of indicator necroptosis proteins like RIPK3, MLKL, RIPK1, and HMGB1 by enzyme-linked immunosorbent assay (ELISA). On this basis, a multiple logistic (regression) classification for the prediction of severe COVID-19 progression was performed.

Results: We found significantly elevated RIPK3, MLKL, HMGB1, and RIPK1 levels in COVID-19 patients admitted to the ICU compared to healthy controls throughout the ongoing disease, indicating necroptotic processes. Above all, with combined measurements of RIPK3 and HMGB1 plasma levels, we were able to time-independently predict COVID-19 severity with $84 \%$ accuracy, $90 \%$ sensitivity, and $76 \%$ specificity.

Conclusion: We suggest that HMGB1 and RIPK3 are potential biomarkers to identify high-risk COVID-19 patients and developed a classifier for COVID-19 severity.

\section{Introduction}

Coronavirus disease 2019 (COVID-19) is the most challenging pandemic in recent human history. In November 2021, the World Health Organization reported 249.743.428 cases of COVID-19 with 5.047 .652 deaths globally [1]. It is crucial for the disease outcome and appropriate treatment to develop a method to determine the exact point of COVID-19 exacerbation. Patients suffering from critical COVID-19 often present with respiratory failure as well as features of sepsis, such as coagulopathy, lymphopenia, and high plasma levels of pro-inflammatory cytokines [2].

In various comparable non-COVID-19-related inflammatory diseases, it is already established that the receptor-interacting serine/threonine-protein kinase 1 and 3 (RIPK1 and RIPK3), as well as the mixed lineage kinase domain-like pseudokinase (MLKL), are associated with disease progression as important regulators of necroptotic cell death [3, 4]. For example, the examination of lung tissue sections in H7N9 virus infection, in which acute respiratory distress syndrome (ARDS) was the main cause of death, showed significantly higher RIPK1, RIPK3, phospho-RIPK3, MLKL, and phospho-MLKL protein levels [5]. These data suggest that severe H7N9 infection is associated with necroptosis of the lung epithelium which contributes to ARDS. This hypothesis is supported by results that showed significantly increased RIPK3 levels not only in the plasma of ARDS patients but also in bronchoalveolar lavage fluid [6]. Furthermore, elevated RIPK3 levels in the plasma of patients with severe sepsis or septic shock also indicate that the RIPK3 signaling pathway is activated under septic conditions [7]. Necroptosis, also referred to as RIPK3-dependent necrosis, is executed by phosphorylated and activated RIPK1 and RIPK3, which form a complex known as the necrosome [8-10]. Subsequently, the effector molecule MLKL is phosphorylated, enabling it to oligomerize and migrate to the cell membrane, leading to the release of damage-associated molecular patterns (DAMPs), cell rupture, and lytic cell death [11]. This promotes cytokine production and an excessive immune response [12]. High-mobility group box 1 (HMGB1), considered as one of the most relevant DAMPs released by necroptotic cells, usually binds to DNA as well as chromatin and exerts its function in chromatin modification and DNA repair [13-18]. When released during inflammatory cell death, HMGB1 triggers immunological processes, inducing recruitment of immune cells and expression as well as the release of pro-inflammatory cytokines (interleukin 6 (IL-6); IL-1 $\beta$; tumor necrosis factor-a (TNF-a)), as similarly described in COVID-19 [13, 19-21]. Extracellular HMGB1 is furthermore capable of forming complexes with cytokines amplifying hyperinflammation [22, 23]. Moreover, high serum HMGB1 levels in non-COVID-19 patients were linked to fatal ARDS [24] . Besides, reactive oxygen species (ROS) production is associated with necroptosis, and mitochondrial ROS (mtROS) production also plays a crucial role in peripheral lymphocytes in severe disease conditions [25-27].

Against this background, we decided to conduct a close monitoring of plasma levels of the necroptosis-related proteins RIPK3, MLKL, RIPK1, and the DAMP HMGB1 in COVID-19 patients throughout intensive care unit (ICU) stay. The current single-center cohort study aims to investigate the prognostic potential of RIPK3, MLKL, HMGB1, and RIPK1 in COVID-19 progression as feasible biomarkers. Using long-term measurement data, we were able to build a classifier that predicts COVID-19 exacerbation independently of time. In addition, we analyzed cell death and mtROS in peripheral leukocytes of ICU COVID-19 patients in single measurements, to verify if these parameters differ in COVID-19 patients as shown before in severe disease conditions, e.g. sepsis patients [27].

\section{Materials And Methods}

\section{COVID-19 cohort}

This is a prospective single-center cohort study of 46 COVID-19 patients ( $\geq 18$-years) who were admitted to the ICU of the University Hospital Frankfurt am Main, Germany, between June 2020 and January 2021. During ICU stay, blood samples were obtained daily at 8 a.m. from admission until ICU discharge. Inflammatory parameters including C-reactive protein (CRP), IL-6, procalcitonin (PCT), lactate dehydrogenase (LDH), and peripheral leukocyte count were obtained daily at 4 a.m. and measured by the hospital's central laboratory and compared to the hospital's central

Page 2/18 
laboratory's threshold levels (CRP: 0.5 mg/dl, IL-6: 7 pg/ml, PCT: 0.5 ng/ml, LDH: 248 U/l, peripheral leukocyte count: 10.41 /nl). Control samples were drawn from 15 non-severe acute respiratory syndrome coronavirus 2 (SARS-CoV-2)-infected healthy donors ( $\geq 18$-years) to compare healthy physiological conditions to COVID-19. The study was conducted in compliance with good clinical practice and current guidelines. Intubation was considered in patients with COVID-19 and severe hypoxemia $\left(\mathrm{PaO}_{2} / \mathrm{FiO}_{2}<150 \mathrm{mmHg}\right)$ and respiratory rates $>30 / \mathrm{min} \mathrm{A} \mathrm{PaO} / \mathrm{FiO}{ }_{2}$ of $<100 \mathrm{mmHg}$ in two consecutive measurements was an indication to perform mechanical ventilation, according to the German guideline [28]. Based on this, it was feasible to distinguish between patients with severe and moderate COVID-19 according to their requirement for intubation throughout their ICU stay. Patients were transferred to a normal ward if their oxygen requirement was $<6 \mathrm{l} / \mathrm{min}$ and their $\mathrm{SpO}_{2}>90 \%$. Patients who were not mechanically ventilated due to patient will $(n=3)$, despite indication, were also assigned to the group of patients with severe COVID-19. The time of symptom onset was specified by the patient.

\section{Plasma preparation and measurement}

Whole blood samples were drawn into citrate tubes (SARSTEDT S Monovetten, Nümbrecht, Germany, Citrat 3,13\%). Samples were centrifuged for 10 minutes at $2000 \mathrm{~g}$ and plasma was stored at $-80^{\circ} \mathrm{C}$ until further processing.

Enzyme-linked immunosorbent assays (ELISAs) (RIPK3 - CSB-EL019737-HU Cusabio, Wuhan, China; RIPK1 - MBS 9137722; MLKL - MBS 9137385, MyBioSource, San Diego, USA; RIPK1 - HEE640Hu, Cloud-Clone Corp., Katy, USA; MLKL - OKEH03401, Aviva Systems Biology, San Diego, USA; HMGB1 - NBP2-62766, Novus Biologicals, Toronto, Canada) were used to determine the plasma concentration of RIPK3, RIPK1, MLKL, and HMGB1.

\section{Statistical analysis}

Statistical analyses were carried out with GraphPad Prism version 7.0 (GraphPad Software Inc., San Diego, CA, USA) and R v4.0.3 (R Foundation for Statistical Computing, Vienna, Austria) [29]. Descriptive variables were calculated using means with standard deviation (SD); medians and interquartile ranges (IQRs, P25\%-P75\%), as well as counts and percentages. For continuous variables, two-tailed Student's t- or Mann-Whitney U tests were performed. ANOVAs with Tukey's post hoc test for multiple comparisons or Kruskal-Wallis tests with Dunn's post hoc test for multiple comparisons were used to examine more than two groups. Adjusted p-values from post hoc tests were indicated ( $p_{a d j}$ ). For categorical data, Fisher's exact test was performed. Principal Component Analysis (PCA) was performed to investigate the relevance of the measurement parameters and their correlations. A $p$-value $<0.05$ was considered statistically significant $\left({ }^{*} p<0.05 ;{ }^{* *} p<0.01 ;{ }^{* \star *} p<0.001\right)$.

\section{Multiple logistic (regression) classifier}

A multiple logistic regression analysis was performed including 28 patients with severe COVID-19, defined by the requirement for mechanical ventilation at the time of the respective blood collection, and 18 patients with moderate COVID-19. The COVID-19 cohort was censored ( $0=$ ICU discharge and $1=$ death). The day of the censoring event was labeled as day E and chosen as a reference point. Data from the days $\mathrm{E}$ to $\mathrm{E}-3 \mathrm{w}$ were used for training and testing the model. The remaining data up to E-7 were used as complementary validation data. The labeled data were split randomly into a training (70\%) and a test set (30\%). Each set contained binary class information about the patients' severity status (moderate $=0$ and severe=1) as well as the quantitative measurements of plasma RIPK3, MLKL, HMGB1, and RIPK1 levels.

Different models were trained and evaluated, including single or combined variables. All models were calculated using a generalized linear model (GLM) of the binomial family to find a classifier (caret package) [30]. A 10-fold cross-validation was performed to exclude a subsample bias and prevent the model from being overfitted. Each model was calculated as a regular logistic regression to compare the relative goodness-of-fit with Akaike's information criterion (AIC). The ideal predictors for the classifier were selected by evaluation of the classification performance indicators, e.g., predictors with the highest accuracy and the lowest AIC after cross-validation were chosen. The final classifier was built with training data that showed mean accuracies $>90 \%$ on days E to E-3. This model was tested with the separated validation set to determine the overall predictive quality of the classifier, e.g., with general performance parameters (Accuracy, Sensitivity, and Specificity). The odds and Odds Ratio (OR) of the predictor variables were determined from the coefficients of the final regression model. Finally, multiple logistic regression models were performed using measurements of RIPK3, HMGB1, CRP, IL-6, PCT, LDH, and peripheral leukocyte count including a training and a test set.

\section{Results}

\section{Demographic characteristics and laboratory parameters}

Between June 2020 and January 2021, we considered 46 COVID-19 patients admitted to the ICU, of whom 28 patients showed a moderate and 18 patients a severe COVID-19 progression during ICU stay. Overall, ICU admission occurred on day seven (4-11) after symptom onset. Patients with severe COVID-19 were older ( $p=0.033)$, showed extended ICU stay $(p<0.001)$, and increased mortality rate $(p<0.001)$ compared to patients with moderate COVID-19 (Table 1). The median survival time after ICU admission in patients with severe COVID-19 was 17 (15-35) days. Of all investigated comorbidities, we found a significantly increased rate of arterial hypertension in patients with severe compared to moderate COVID-19 $(p=0.016)$ (Table 1). 
We also examined median levels of the patients' laboratory parameters during ICU stay, reflecting immunologic and inflammatory abnormalities (Table 1). Median levels of CRP ( $p<0.001)$, IL-6 ( $<<0.001)$, PCT $(p<0.001)$, LDH $(p=0.033)$, and peripheral leukocyte count ( $<<0.001)$ were significantly higher in patients with severe compared to moderate COVID-19. Furthermore, CRP (95.7\%), IL-6 (87\%), and LDH (87\%) median levels in these patients were elevated compared to the hospital's central laboratory's threshold. Moreover, elevated peripheral leukocyte counts (55.6\% versus $14.3 \%$; $p=0.007$ ) and PCT levels (61.1\% versus $10.7 \%$; $\mathrm{p}=0.001)$ were significantly more common in patients with severe compared to moderate COVID-19.

\section{Prolonged measurements of plasma RIPK3, MLKL, HMGB1, and RIPK1 in COVID-19 intensive care patients}

During ICU stay, we measured RIPK3, MLKL, HMGB1, and RIPK1 levels daily in COVID-19 patients from admission until discharge or death (Fig. 1). In patients with moderate as well as severe COVID-19, we found significantly higher levels of RIPK3 (Fig. 1a), MLKL (Fig. 1b), HMGB1 (Fig. 1c), and RIPK1 (Fig. 1d) compared to healthy controls (the median length of ICU stay in patients with moderate COVID-19 was 6 (4-8) days and with severe COVID-19 was 16 (13.5-21.8) days).

Additionally, we examined these measurements with symptom onset as a baseline (Fig. S1).

The PCA, including RIPK3, MLKL, HMGB1, and RIPK1, revealed a positive association between HMGB1 and MLKL (Fig. 2a,c,d). Combined measurements of RIPK3, MLKL, HMGB1, and RIPK1 segregated COVID-19 patients from healthy controls, whereas an overlap between patients with severe COVID-19 and patients with moderate COVID-19 remained in a two-dimensional PCA scatterplot (Fig. 2b). Therefore, we next examined RIPK3, MLKL, HMGB1, and RIPK1 plasma levels individually and in various combinations to predict disease severity more accurately.

\section{Prediction of severe COVID-19 progression with a multiple logistic (regression) classifier: model selection}

With randomly selected training data from the day of the censoring event E (ICU discharge or death) to E-3 (three days before the censoring event), we evaluated models with different predictor combinations in a time-independent manner (Table 2). Although a model consisting of HMGB1, RIPK3, and RIPK1 and a model consisting of solely RIPK3 achieved better accuracies with 78\%, their fits and thus model qualities (Akaike's information criterion (AIC)) were worse, resulting in a model with HMGB1 and RIPK3 with a marginally superior fit (AIC=65.91), pointing to a simpler and, therefore, more applicable model. While this was an improvement towards the other models, a slightly lower classification accuracy AcC $_{\mathrm{HMGB} 1+\mathrm{RIPK} 3}=77 \% \mathrm{Was}$ achieved. However, this model required only two measurements, opposed to three.

To further evaluate on which days plasma RIPK3, MLKL, HMGB1, and RIPK1 levels distinguished best between severe and moderate COVID-19 progression, we looked at individual days and markers backward from the censoring event (E). On days E-3 until the event, HMGB1 (Fig. 3a-d, third column) and on days E-3 and E-1, RIPK3 (Fig. 3b,d, first column) plasma levels were significantly elevated in patients with severe compared to those with moderate COVID-19. In contrast, RIPK1 and MLKL levels did not differ significantly between severe and moderate COVID-19 (Fig. 3a-d, second and fourth column) and were therefore not included in our further analysis. Consequently, plasma RIPK3 and HMGB1 were selected for further evaluation.

\section{Evaluation of the discriminatory ability of combined RIPK3 and HMGB1 plasma levels for building the COVID-19 severity classifier}

To organize data for training and testing the selected model, combined RIPK3 and HMGB1 plasma levels are viewed backward from the censoring event (E). Table 3 shows the classification performance of predicting COVID-19 progression using the training and test data. The days starting from day $\mathrm{E}$ to $\mathrm{E}-3$ were analyzed separately. In the training data, $\mathrm{E}, \mathrm{E}-1$, and $\mathrm{E}-3$ achieved accuracies of $100 \%\left(\mathrm{Cl}_{95 \%, \mathrm{E}}[78 ; 100]\right),(\mathrm{Cl} 95 \%, \mathrm{E}-1$ [ $[79 ; 100])$, and $\left(\mathrm{Cl}_{95 \%, \mathrm{E}-3}[79 ; 100]\right)$, as well as high sensitivity and specificity $(100 \%)$, indicating that the combined measurement of HMGB1 and RIPK3 levels on these days discriminated well between patients with moderate and severe COVID-19. On day E-2, the accuracy was lower $(82 \%)(\mathrm{Cl} 95 \%, \mathrm{E}-2[57 ; 96])$, mainly due to a loss in specificity. In the test data, the event day was classified at $76 \%\left(\mathrm{Cl}_{95 \%, \mathrm{E}, \text { test }}[61 ; 87]\right)$ and day E-1 was classified lowest at $72 \%$ accuracy $\left(\mathrm{Cl}_{95 \%, \mathrm{E}-1, \text { test }}[57 ; 84]\right)$, while day $\mathrm{E}-2$ reached $78 \%\left(\mathrm{Cl}_{95 \%, \mathrm{E}-2, \text { test }}[64 ; 89]\right)$. On day $\mathrm{E}-3$, the test data were best classified with an accuracy of $83 \%$ $\left(\mathrm{Cl}_{95 \%, \mathrm{E}-3, \text { test }}[69 ; 92]\right)$.

The most stable and optimal results in discriminating between patients with moderate and severe COVID-19 were found to be days E-1 and E-3, as indicated by significantly higher RIPK3 and HMGB1 plasma levels (Fig. 3b,d, first and third column) as well as by the performance of the training and test data using combined RIPK3 and HMGB1 measurements from these days (Table 3). Therefore, HMGB1 and RIPK3 plasma levels from these days were used in building the final classifier.

\section{Prediction of severe COVID-19 progression with combined RIPK3 and HMGB1 measurements}

Table 4 shows the performance of the final classifier with the training, test, and validation data. The overall accuracies of discriminating between a moderate and severe COVID-19 progression were high (>83\%). The fraction of false positives and false negatives was low, resulting in specificity and sensitivity levels $>74 \%$. The test set reached $83 \%$ accuracy as well as $89 \%$ sensitivity and $74 \%$ specificity (Fig. $4 \mathrm{~b}$ ), which was exceeded by the validation set using data from up to day E-7 (excluding E-1 and E-3) with $84 \%$ accuracy, $90 \%$ sensitivity, and $76 \%$ specificity (Fig. 4c). This was particularly accurate up to 6 days before the censoring event (Fig. 4d). 
Also, RIPK3 plasma levels of patients with moderate COVID-19 approached the healthy control levels before ICU discharge (Fig. 4e). Notably, HMGB1 plasma levels indicated significant differences between patients with moderate and severe COVID-19 at a very early stage (E-8) (Fig. 4f). Therefore, the combination of circulating levels of RIPK3 and HMGB1 can be used to time-independently classify COVID-19 patients admitted to the ICU into potential disease severity states (Fig. 4a-c).

\section{The odds of changing COVID-19 severity based on RIPK3 and HMGB1 levels}

To further estimate these findings, a logistic regression model was calculated with the full data over the entire observation period of plasma RIPK3 and HMGB1 levels as independent variables and disease progression of COVID-19 as the dependent variable. With this model, the odds of changing the disease severity state were estimated (Table 5).

For every unit change in RIPK3, the log-odds of disease severity change from moderate to severe were increased by 0.305 (Odds Ratio (OR) $=1.36$ $\left.\left(\mathrm{Cl}_{95 \%}[1.22 ; 1.52]\right)\right)$. Similarly, every unit change in HMGB1 increased the log-odds by $0.006\left(\mathrm{OR}=1.006\left(\mathrm{Cl}_{95 \%}[1.004 ; 1.007]\right)\right)$.

\section{Model comparison for the prediction of severe COVID-19 progression using multiple inflammatory variables}

To compare the predictive power of established inflammatory markers as well as RIPK3 and HMGB1, we additionally performed a multiple logistic regression model including measurements of RIPK3, HMGB1, CRP, IL-6, PCT, LDH, and peripheral leukocyte count. Interestingly, in the training set, the combination of measured RIPK3, HMGB1, and PCT levels reached the highest accuracy (93\%). In order to be able to represent the plot in two dimensions, with one variable on the x-axis and one on the $y$-axis, we chose a model with two measurements from our potential biomarkers. In fact, the combination of RIPK3 and HMGB1 levels discriminated best between moderate and severe COVID-19 progression with an accuracy of $86 \%$ in the training set (Table 6). In the test set, both models performed similarly, with an accuracy of $83.7 \%$ (Table 7). Therefore, plasma RIPK3 and HMGB1 are the most suitable candidates for predicting COVID-19 severity.

\section{Discussion}

In this study, plasma RIPK3, MLKL, HMGB1, and RIPK1 levels of COVID-19 patients are obtained in daily-assessed measurements throughout the whole ICU stay. Based on these data, we developed for the first time a classifier built on RIPK3 and HMGB1 as potential biomarkers to discriminate between moderate and severe COVID-19 progression after ICU admission with an accuracy of $84 \%$. Several independent lines of evidence support this conclusion.

First, COVID-19 intensive care patients showed continuously significantly higher plasma RIPK3 levels than healthy controls throughout their ICU stay, strongly indicating ongoing RIPK3-dependent necroptosis. In addition to previous investigations that considered RIPK3 levels at single time points [31, 32], we revealed in our prolonged study that patients with severe COVID-19 possess higher RIPK3 plasma levels in a time-dependent manner. Also, patients with moderate COVID-19 showed decreasing RIPK3 levels, corresponding to their recovery.

Second, we observed significant long-term elevations of HMGB1 in COVID-19 intensive care patients compared to healthy controls. Elevations of HMGB1 levels were associated with the requirement for mechanical ventilation and fatal outcome, as also demonstrated in our supplemental data and previous studies [33,34]. Notably, we also revealed significant elevations of plasma HMGB1 corresponding to severe COVID-19 progression in a disease-dependent time course. Chen et al. observed an association between exogenous human HMGB1 and stimulated angiotensin-converting enzyme 2 (ACE2) expression as an entry receptor for SARS-CoV-2 in cultured human lung epithelial cells, indicating a feedback loop that possibly worsens patients' outcomes [33]. RIPK3 and extracellular HMGB1 also contribute to endothelial dysfunction and loss of barrier integrity, considered to be involved in COVID-19 pathology [6,35-37]. Since high extracellular levels of HMGB1 are particularly harmful, our results provide evidence for HMGB1 as a potential drug target in COVID-19, as has been successfully demonstrated for IL-6 signaling [38].

Third, we found that plasma MLKL and RIPK1 levels were tendentially higher in COVID-19 ICU patients compared to healthy controls, indicating an involvement of necroptosis in COVID-19 pathology. Accordingly, upregulation of phosphorylated MLKL was detected in lung tissue of SARS-CoV-2-infected mice and post mortem human lungs. In vitro, MLKL and RIPK3 contributed to cell death induction, as well as cytokine and DAMP release in SARS-CoV-2-infected cells, reinforcing our findings in COVID-19 patients in the ICU [39]. Moreover, phosphorylated and thus activated RIPK1 was detected in pharyngeal epithelial cells of COVID-19 patients, and since respiratory tissues appeared to be a prominent sink for RIPK1 in COVID-19, its interaction with SARS-CoV-2 components is hypothesized [40,41]. However, MLKL and RIPK1 did not contribute significantly to COVID-19 severity and were therefore not included in the final classifier.

In addition to our prolonged study investigating kinetic variations, our single measurements of RIPK3, MLKL, HMGB1, and RIPK1 supported our hypothesis that necroptosis plays a role in COVID-19, as described in our supplemental data. In this cohort, we also observed a loss of viable peripheral leukocytes in every examined cell subpopulation according to disease severity, particularly in patients receiving extracorporeal membrane oxygenation (ECMO), however, given that there were only 6 patients with this treatment, these results should be interpreted carefully.

To our knowledge, we are the first to perform mtROS measurements using flow cytometry in whole blood samples of COVID-19 patients; therefore, there is still a lack of comparative studies. Other studies were carried out on cell cultures treated with plasma from COVID-19 patients or with single 
viral components (open reading frame 3a (ORF-3a) or the SARS-CoV-2 spike protein), as well as SARS-CoV-2-infected monocytes in vitro and respiratory samples (sputum/ Bronchoalveolar lavage (BAL)) of COVID-19 patients [42-46]. Nevertheless, it is important to mention these studies, but comparisons should be interpreted with caution. We show in our supplemental data that peripheral leukocytes of our COVID-19 cohort with single measurements had significantly lower levels of mtROS compared to healthy controls.

In COVID-19 patients, cytokines like TNF- $a$ and IL- 6 are mainly released by non-circulating cells, indicating pathological processes in infected or damaged tissue [32, 47]. We therefore assume that RIPK3, MLKL, HMGB1, and RIPK1 are released, for instance, by virus-infected lung tissue cells, damaged vascular endothelial cells, or activated immune cells recruited to the site of infection $[5,6,39,48-50]$.

As patients were admitted to the ICU at different disease stages, data from the first day after ICU admission would provide limited information. Therefore, we took data from time points when the disease progression was already clear to build our model using plasma RIPK3 and HMGB1 levels and thereby reduced the variability that resulted from admission to the ICU at different COVID-19 stages. In everyday clinical practice, it is often not possible to predict whether a patient is close to or long before death or ICU discharge. The classifier model avoids this problem with RIPK3 and HMGB1 as promising biomarkers in COVID-19.

This study has several limitations. The timing of mechanical ventilation is a subjective outcome. However, differentiation of severity is possible because, once patients have the indication for intubation, spontaneous breathing and non-invasive ventilation are no longer sufficient and a definite state of disease progression has been reached. Since we intended to examine COVID-19 patients over a prolonged period, we decided to consider the requirement for intubation as a distinction between a moderate and severe COVID-19 progression for the study design. We cannot completely exclude an additional impact of the intubation status on plasma levels of RIPK3, MLKL, HMGB1, and RIPK1.

We are aware that measurements of 46 patients must be considered carefully, but regarding the number of blood samples received daily over a longer period of time, the study size is unusually extensive, in particular, compared to other single-center studies.

Moreover, to further explore the disease mechanisms indicated by this study, we suggest additional investigations on necroptosis markers, such as studies on other COVID-19 progressions and stages which we could not take into account e.g., non-hospitalized patients, or patients with post-COVID-19 syndrome. Finally, our data needs to be confirmed in further longitudinal clinical studies with independent cohorts of COVID-19 patients before implementation in clinical algorithms can be considered.

\section{Conclusion}

Our classifier with RIPK3 and HMGB1 as promising biomarkers in COVID-19 could help to timely identify future patients who require more intensive monitoring and benefit from maximized immunomodulatory therapy after ICU admission [38, 51]. This model is simple and more accurate than models that, in addition to RIPK3 and HMGB1 plasma levels, considered inflammatory markers such as CRP, IL-6, PCT, LDH, and peripheral leukocyte count.

\section{Abbreviations}

$\begin{array}{ll}\text { Acc } & \text { accuracy } \\ \text { ARDS } & \text { acute respiratory distress syndrome } \\ \text { AIC } & \text { Akaike's Information criterion } \\ \text { ACE2 } & \text { angiotensin-converting enzyme 2 } \\ \text { BAL } & \text { Bronchoalveolar lavage } \\ \text { COPD } & \text { chronic obstructive pulmonary disease } \\ \text { COVID-19 } & \text { coronavirus disease 2019 } \\ \text { CRP } & \text { C-reactive protein } \\ \text { DAMPs } & \text { damage-associated molecular patterns } \\ \text { ECMO } & \text { Extracorporeal membrane oxygenation } \\ \text { ELISA } & \text { enzyme-linked immunosorbent assay } \\ \text { GLM } & \text { generalized linear model } \\ \text { HMGB1 } & \text { high-mobility group box 1 }\end{array}$


procalcitonin

RIPK3 receptor-interacting serine/threonine-protein kinase 3

RIPK1

receptor-interacting serine/threonine-protein kinases 1

ROS

reactive oxygen species

$\beta$ regression coefficient severe acute respiratory syndrome coronavirus 2

\section{Declarations}

\section{Ethics approval and consent to participate}

The study was performed in accordance with the Declaration of Helsinki. Approval from the local ethics committee was obtained before the study was conducted (reference \#20-643, \#20-982) and a waiver regarding the requirement of written informed consent from COVID-19 patients was authorized. All participants of the control group provided written informed consent.

\section{Consent for publication}

All authors critically revised and approved the manuscript.

\section{Data and materials availability}

All data are available in the main text or the supplementary materials.

\section{Author contributions}

Contribution: U.H. designed research; K.R. performed experiments; K.R. and H.N. collected data; K.R., U.H., and S.T. performed the analyses; K.R., U.H., S.T., and S.C. wrote the manuscript with input from H.N., E.A., A.v.K., and K.Z.

\section{Competing interests}

KZ: The Department of Anaesthesiology, Intensive Care Medicine \& Pain Therapy of the University Hospital Frankfurt received support from B. Braun Melsungen, CSL Behring, Fresenius Kabi, and Vifor Pharma for the implementation of Frankfurt's Patient Blood Management program and KZ received honoraria for scientific lectures and ad board meeting within the last 3 years from CSL Behring, GE Healthcare, Edwards, Haemonetics, implatcast $\mathrm{GmbH}$, med Update $\mathrm{GmbH}$, Pharmacosmos and Vifor Pharma. The remaining authors declare no potential conflicts of interest.

\section{Funding}

Supported by the Goethe Coronavirus Fund of the Goethe University \& University Hospital Frankfurt 


\section{References}

1. WHO Coronavirus (COVID-19) Dashboard I WHO Coronavirus (COVID-19) Dashboard With Vaccination Data. 08.11.2021. https://covid19.who.int/. Accessed 8 Nov 2021.

2. Huang C, Wang Y, Li X, Ren L, Zhao J, Hu Y, et al. Clinical features of patients infected with 2019 novel coronavirus in Wuhan, China. The Lancet. 2020;395:497-506. doi:10.1016/S0140-6736(20)30183-5.

3. Shashaty MGS, Reilly JP, Faust HE, Forker CM, Ittner CAG, Zhang PX, et al. Plasma receptor interacting protein kinase-3 levels are associated with acute respiratory distress syndrome in sepsis and trauma: a cohort study. Crit Care. 2019;23:235. doi:10.1186/s13054-019-2482-x.

4. Vucur M, Roderburg C, Kaiser L, Schneider AT, Roy S, Loosen SH, et al. Elevated Serum Levels of Mixed Lineage Kinase Domain-Like Protein Predict Survival of Patients during Intensive Care Unit Treatment. Dis Markers. 2018;2018:1983421. doi:10.1155/2018/1983421.

5. Qin C, Sai X, Qian X, Wu Y, Zou L, Wang H, et al. Close Relationship between clAP2 and Human ARDS Induced by Severe H7N9 Infection. Biomed Res Int. 2019;2019:2121357. doi:10.1155/2019/2121357.

6. Yu X, Mao M, Liu X, Shen T, Li T, Yu H, et al. A cytosolic heat shock protein 90 and co-chaperone p23 complex activates RIPK3/MLKL during necroptosis of endothelial cells in acute respiratory distress syndrome. J Mol Med (Berl). 2020;98:569-83. doi:10.1007/s00109-020-01886-y.

7. Ma KC, Schenck EJ, Siempos II, Cloonan SM, Finkelsztein EJ, Pabon MA, et al. Circulating RIPK3 levels are associated with mortality and organ failure during critical illness. JCI Insight 2018. doi:10.1172/jci.insight.99692.

8. Cho YS, Challa S, Moquin D, Genga R, Ray TD, Guildford M, Chan FK-M. Phosphorylation-driven assembly of the RIP1-RIP3 complex regulates programmed necrosis and virus-induced inflammation. Cell. 2009;137:1112-23. doi:10.1016/j.cell.2009.05.037.

9. Li J, McQuade T, Siemer AB, Napetschnig J, Moriwaki K, Hsiao Y-S, et al. The RIP1/RIP3 necrosome forms a functional amyloid signaling complex required for programmed necrosis. Cell. 2012;150:339-50. doi:10.1016/j.cell.2012.06.019.

10. He S, Wang L, Miao L, Wang T, Du F, Zhao L, Wang X. Receptor interacting protein kinase-3 determines cellular necrotic response to TNF-alpha. Cell. 2009;137:1100-11. doi:10.1016/j.cell.2009.05.021.

11. Wang H, Sun L, Su L, Rizo J, Liu L, Wang L-F, et al. Mixed lineage kinase domain-like protein MLKL causes necrotic membrane disruption upon phosphorylation by RIP3. Molecular Cell. 2014;54:133-46. doi:10.1016/j.molcel.2014.03.003.

12. Duprez L, Takahashi N, van Hauwermeiren F, Vandendriessche B, Goossens V, Vanden Berghe T, et al. RIP kinase-dependent necrosis drives lethal systemic inflammatory response syndrome. Immunity. 2011;35:908-18. doi:10.1016/j.immuni.2011.09.020.

13. Scaffidi P, Misteli T, Bianchi ME. Release of chromatin protein HMGB1 by necrotic cells triggers inflammation. Nature. 2002;418:191-5. doi:10.1038/nature00858.

14. Simpson J, Loh Z, Ullah MA, Lynch JP, Werder RB, Collinson N, et al. Respiratory Syncytial Virus Infection Promotes Necroptosis and HMGB1 Release by Airway Epithelial Cells. Am J Respir Crit Care Med. 2020;201:1358-71. doi:10.1164/rccm.201906-11490C.

15. Qing DY, Conegliano D, Shashaty MGS, Seo J, Reilly JP, Worthen GS, et al. Red blood cells induce necroptosis of lung endothelial cells and increase susceptibility to lung inflammation. Am J Respir Crit Care Med. 2014;190:1243-54. doi:10.1164/rccm.201406-10950C.

16. Bianchi ME, Beltrame M, Paonessa G. Specific recognition of cruciform DNA by nuclear protein HMG1. Science. 1989;243:1056-9. doi:10.1126/science.2922595.

17. Catez F, Yang H, Tracey KJ, Reeves R, Misteli T, Bustin M. Network of dynamic interactions between histone H1 and high-mobility-group proteins in chromatin. Mol Cell Biol. 2004;24:4321-8. doi:10.1128/mcb.24.10.4321-4328.2004.

18. Lange SS, Mitchell DL, Vasquez KM. High mobility group protein B1 enhances DNA repair and chromatin modification after DNA damage. Proc Natl Acad Sci U S A. 2008;105:10320-5. doi:10.1073/pnas.0803181105.

19. Venereau E, Casalgrandi M, Schiraldi M, Antoine DJ, Cattaneo A, Marchis F de, et al. Mutually exclusive redox forms of HMGB1 promote cell recruitment or proinflammatory cytokine release. J Exp Med. 2012;209:1519-28. doi:10.1084/jem.20120189.

20. Andersson U, Wang H, Palmblad K, Aveberger AC, Bloom O, Erlandsson-Harris H, et al. High mobility group 1 protein (HMG-1) stimulates proinflammatory cytokine synthesis in human monocytes. J Exp Med. 2000;192:565-70. doi:10.1084/jem.192.4.565.

21. Del Valle DM, Kim-Schulze S, Huang H-H, Beckmann ND, Nirenberg S, Wang B, et al. An inflammatory cytokine signature predicts COVID-19 severity and survival. Nat Med. 2020;26:1636-43. doi:10.1038/s41591-020-1051-9.

Page 8/18 
22. Coleman LG, Maile R, Jones SW, Cairns BA, Crews FT. HMGB1/IL-1 $\beta$ complexes in plasma microvesicles modulate immune responses to burn injury. PLoS One. 2018;13:e0195335. doi:10.1371/journal.pone.0195335.

23. Hreggvidsdottir HS, Ostberg T, Wähämaa $H$, Schierbeck $H$, Aveberger $A-C$, Klevenvall $L$, et al. The alarmin HMGB1 acts in synergy with endogenous and exogenous danger signals to promote inflammation. J Leukoc Biol. 2009;86:655-62. doi:10.1189/jlb.0908548.

24. Nakamura T, Sato E, Fujiwara N, Kawagoe Y, Maeda S, Yamagishi S. Increased levels of soluble receptor for advanced glycation end products (sRAGE) and high mobility group box 1 (HMGB1) are associated with death in patients with acute respiratory distress syndrome. Clinical Biochemistry. 2011;44:601-4. doi:10.1016/j.clinbiochem.2010.12.014.

25. Schenk B, Fulda S. Reactive oxygen species regulate Smac mimetic/TNFa-induced necroptotic signaling and cell death. Oncogene. 2015;34:5796-806. doi:10.1038/onc.2015.35.

26. Zhang D-W, Shao J, Lin J, Zhang N, Lu B-J, Lin S-C, et al. RIP3, an energy metabolism regulator that switches TNF-induced cell death from apoptosis to necrosis. Science. 2009;325:332-6. doi:10.1126/science.1172308.

27. Ayala JC, Grismaldo A, Aristizabal-Pachon AF, Mikhaylenko EV, Nikolenko VN, Mikhaleva LM, et al. Mitochondrial Dysfunction In Intensive Care Unit Patients. Current pharmaceutical design 2020. doi:10.2174/1381612826666201207112931.

28. Kluge S, Janssens U, Welte T, Weber-Carstens S, Schälte G, Spinner CD, et al. 113-001LGI_S3_Empfehlungen-zur-stationaeren-Therapie-vonPatienten-mit-COVID-19_2021-05.

29. R: The R Project for Statistical Computing.

30. Max Kuhn (2020). caret: Classification and Regression Training. R package version 6.0-86.

31. Nakamura H, Kinjo T, Arakaki W, Miyagi K, Tateyama M, Fujita J. Serum levels of receptor-interacting protein kinase-3 in patients with COVID-19. Crit Care. 2020;24:484. doi:10.1186/s13054-020-03209-6.

32. Hadjadj J, Yatim N, Barnabei L, Corneau A, Boussier J, Smith N, et al. Impaired type I interferon activity and inflammatory responses in severe COVID-19 patients. Science. 2020;369:718-24. doi:10.1126/science.abc6027.

33. Chen R, Huang Y, Quan J, Liu J, Wang H, Billiar TR, et al. HMGB1 as a potential biomarker and therapeutic target for severe COVID-19. Heliyon. 2020;6:e05672. doi:10.1016/j.heliyon.2020.e05672.

34. Chen L, Long X, Xu Q, Tan J, Wang G, Cao Y, et al. Elevated serum levels of S100A8/A9 and HMGB1 at hospital admission are correlated with inferior clinical outcomes in COVID-19 patients. Cell Mol Immunol. 2020;17:992-4. doi:10.1038/s41423-020-0492-X.

35. Wolfson RK, Chiang ET, Garcia JGN. HMGB1 induces human lung endothelial cell cytoskeletal rearrangement and barrier disruption. Microvasc Res. 2011;81:189-97. doi:10.1016/j.mvr.2010.11.010.

36. Varga Z, Flammer AJ, Steiger P, Haberecker M, Andermatt R, Zinkernagel AS, et al. Endothelial cell infection and endotheliitis in COVID-19. The Lancet. 2020;395:1417-8. doi:10.1016/S0140-6736(20)30937-5.

37. Huang W, Liu Y, Li L, Zhang R, Liu W, Wu J, et al. HMGB1 increases permeability of the endothelial cell monolayer via RAGE and Src family tyrosine kinase pathways. Inflammation. 2012;35:350-62. doi:10.1007/s10753-011-9325-5.

38. RECOVERY Collaborative Group. Tocilizumab in patients admitted to hospital with COVID-19 (RECOVERY): a randomised, controlled, open-label, platform trial. The Lancet. 2021;397:1637-45. doi:10.1016/S0140-6736(21)00676-0.

39. Li S, Zhang Y, Guan Z, Li H, Ye M, Chen X, et al. SARS-CoV-2 triggers inflammatory responses and cell death through caspase-8 activation. Signal Transduct Target Ther. 2020;5:235. doi:10.1038/s41392-020-00334-0.

40. Riebeling T, Jamal K, Wilson R, Kolbrink B, Samson-Himmelstjerna FA von, Moerke C, et al. Primidone blocks RIPK1-driven cell death and inflammation. Cell Death Differ. 2020;28:1610-1626. doi:10.1038/s41418-020-00690-y.

41. Feng L, Yin YY, Liu CH, Xu KR, Li QR, Wu JR, Zeng R. Proteome-wide Data Analysis Reveals Tissue-specific Network Associated with SARS-CoV-2 Infection. J Mol Cell Biol. 2021;12:946-57. doi:10.1093/jmcb/mjaa033.

42. Codo AC, Davanzo GG, Monteiro LdB, Souza GF de, Muraro SP, Virgilio-da-Silva JV, et al. Elevated Glucose Levels Favor SARS-CoV-2 Infection and Monocyte Response through a HIF-1a/Glycolysis-Dependent Axis. Cell metabolism. 2020;32:437-446.e5. doi:10.1016/j.cmet.2020.07.007.

43. Miripour ZS, Sarrami-Forooshani R, Sanati H, Makarem J, Taheri MS, Shojaeian F, et al. Real-time diagnosis of reactive oxygen species (ROS) in fresh sputum by electrochemical tracing; correlation between COVID-19 and viral-induced ROS in lung/respiratory epithelium during this pandemic.

Page $9 / 18$ 
Biosens Bioelectron. 2020;165:112435. doi:10.1016/j.bios.2020.112435.

44. Tian M, Liu W, Li X, Zhao P, Shereen MA, Zhu C, et al. HIF-1a promotes SARS-CoV-2 infection and aggravates inflammatory responses to COVID19. Signal Transduct Target Ther. 2021;6:308. doi:10.1038/s41392-021-00726-w.

45. Clough E, Chean KT, Inigo J, Tubbesing KE, Chandra D, Chaves L, et al. Mitochondrial Dynamics in SARS-COV2 Spike Protein Treated Human Microglia: Implications for Neuro-COVID. J Neuroimmune Pharmacol. 2021:1-15. doi:10.1007/s11481-021-10015-6.

46. Andargie TE, Tsuji N, Seifuddin F, Jang MK, Yuen PS, Kong H, et al. Cell-free DNA maps COVID-19 tissue injury and risk of death and can cause tissue injury. JCl Insight 2021. doi:10.1172/jci.insight.147610.

47. Kahn R, Schmidt T, Golestani K, Mossberg A, Gullstrand B, Bengtsson AA, Kahn F. Mismatch between circulating cytokines and spontaneous cytokine production by leukocytes in hyperinflammatory COVID-19. J Leukoc Biol. 2021;109:115-20. doi:10.1002/JLB.5COVBCR0720-310RR.

48. Zhou Z, Ren L, Zhang L, Zhong J, Xiao Y, Jia Z, et al. Heightened Innate Immune Responses in the Respiratory Tract of COVID-19 Patients. Cell Host Microbe. 2020;27:883-890.e2. doi:10.1016/j.chom.2020.04.017.

49. Wang L, Wang T, Li H, Liu Q, Zhang Z, Xie W, et al. Receptor Interacting Protein 3-Mediated Necroptosis Promotes Lipopolysaccharide-Induced Inflammation and Acute Respiratory Distress Syndrome in Mice. PLOS ONE. 2016;11:e0155723. doi:10.1371/journal.pone.0155723.

50. Szabo PA, Dogra P, Gray JI, Wells SB, Connors TJ, Weisberg SP, et al. Longitudinal profiling of respiratory and systemic immune responses reveals myeloid cell-driven lung inflammation in severe COVID-19. Immunity. 2021;54:797-814.e6. doi:10.1016/j.immuni.2021.03.005.

51. Cavalli G, Luca G de, Campochiaro C, Della-Torre E, Ripa M, Canetti D, et al. Interleukin-1 blockade with high-dose anakinra in patients with COVID19, acute respiratory distress syndrome, and hyperinflammation: a retrospective cohort study. The Lancet Rheumatology. 2020;2:e325-e331.

doi:10.1016/S2665-9913(20)30127-2.

\section{Tables}

Table 1.Patient demographics of the COVID-19 cohort.

\begin{tabular}{lcccc}
\hline & Overall & Moderate & Severe & p \\
\hline Total $^{\mathrm{a}}$ & $46(100 \%)$ & $28(60.9 \%)$ & $18(39.1 \%)$ & \\
Gender, female $^{\mathrm{a}}$ & $12(26.1 \%)$ & $8(28.6 \%)$ & $4(22.2 \%)$ & 0.739 \\
Age $^{\mathrm{b}}$, yr & $66.5(49-78)$ & $56(47.8-72)$ & $73.5(65.3-81)$ & 0.033 \\
Bodyweight $^{\mathrm{b}}, \mathrm{kg}$ & $89(78.2-109)$ & $91.5(77.3-111.3)$ & $85(79.1-98.8)$ & 0.596 \\
ICU stay $^{\mathrm{b}}$, d & $8(4.3-16)$ & $6(4-8)$ & $16(13.5-21.8)$ & $<0.001$ \\
Outcome, death $^{\mathrm{a}}$ & $14(30.4 \%)$ & $0(0 \%)$ & $14(77.8 \%)$ & $<0.001$
\end{tabular}

Comorbidity

$\begin{array}{lcccc}\text { Arterial hypertension }^{\mathrm{a}} & 25(54.3 \%) & 11(39.3 \%) & 14(77.8 \%) & 0.016 \\ \text { Diabetes mellitus }^{\mathrm{a}} & 13(28.3 \%) & 5(17.9 \%) & 8(44.4 \%) & 0.092 \\ \text { Adipositas }^{\mathrm{a}} & 15(32.6 \%) & 10(35.7 \%) & 5(27.8 \%) & 0.749 \\ \text { COPD }^{\mathrm{a}} & 6(13 \%) & 2(7.1 \%) & 4(22.2 \%) & 0.191 \\ \text { Asthma bronchiale }^{\mathrm{a}} & 3(6.5 \%) & 2(7.1 \%) & 1(5.6 \%) & 1.000\end{array}$




\begin{tabular}{|c|c|c|c|c|}
\hline $\mathrm{CRP}^{\mathrm{b}}, \mathrm{mg} / \mathrm{dl}$ & $7.6(3.9-14.2)$ & $5.4(3.4-8.8)$ & $13.72(8.9-18.7)$ & 0.001 \\
\hline$>0.5 \mathrm{mg} / \mathrm{dl}^{\mathrm{a}}$ & $44(95.7 \%)$ & $26(92.9 \%)$ & $18(100 \%)$ & 0.513 \\
\hline Leukocyte count ${ }^{\mathrm{b}}, / \mathrm{nl}$ & $8.5(6.4-11.7)$ & $6.7(5.8-8.8)$ & $11.4(9-13.3)$ & $<0.001$ \\
\hline$>10.41 / \mathrm{nl}^{\mathrm{a}}$ & $14(30.4 \%)$ & $4(14.3 \%)$ & $10(55.6 \%)$ & 0.007 \\
\hline IL $-6^{b}, \mathrm{pg} / \mathrm{ml}$ & $35.9(15-111.6)$ & $21.2(8.5-37.8)$ & $124(80.2-207.8)$ & $<0.001$ \\
\hline$>7 \mathrm{pg} / \mathrm{ml}^{\mathrm{a}}$ & $40(87 \%)$ & $22(78.6 \%)$ & $18(100 \%)$ & 0.068 \\
\hline $\mathrm{PCT}^{\mathrm{b}}, \mathrm{ng} / \mathrm{ml}$ & $0.2(0.1-0.7)$ & $0.1(0.1-0.2)$ & $0.7(0.4-2.2)$ & $<0.001$ \\
\hline$>0.5 \mathrm{ng} / \mathrm{ml}^{\mathrm{a}}$ & $14(30.4 \%)$ & $3(10.7 \%)$ & $11(61.1 \%)$ & $<0.001$ \\
\hline $\mathrm{LDH}^{\mathrm{b}}, \mathrm{U} / 1$ & $365.5(315.9-441.8)$ & 352.5 (291.1-399.5) & $429.5(355.1-494)$ & 0.033 \\
\hline$>248 \mathrm{U} / 1^{\mathrm{a}}$ & $40(87 \%)$ & $22(78.6 \%)$ & $18(100 \%)$ & 0.068 \\
\hline
\end{tabular}

Data are presented as ${ }^{\mathrm{a}} \mathrm{n}(\%)$ for categorical variables or ${ }^{\mathrm{b}}$ median (interquartile range) for continuous variables. Patients' laboratory parameters are reported as the respective median of the parameter levels obtained during ICU stay. p-values comparing patients with moderate and severe COVID-19 were calculated with Mann-Whitney U test or Fisher's exact test. Additionally, patients' median laboratory parameter levels were compared to the hospital's central laboratory's threshold levels (CRP: 0.5 mg/dl, IL-6: 7 pg/ml, PCT: 0.5 ng/ml, LDH: 248 U/l, peripheral leukocyte count: 10.41 /nl). Respective quantities in the pathological range were determined and then compared among patients with severe and moderate COVID-19 by Fisher's exact test.

COPD, chronic obstructive pulmonary disease

Table 2. GLM model performances of the training data for prediction of COVID-19 severity.

\begin{tabular}{lll} 
Model & Accuracy (\%) & AlC \\
\hline HMGB1 + RIPK3 & 0.77 & 65.91 \\
\hline HMGB1 + RIPK3 + RIPK1 & 0.78 & 67.88 \\
\hline HMGB1 + RIPK3 + RIPK1 + MLKL & 0.75 & 69.23 \\
\hline HMGB1 & 0.75 & 72.17 \\
\hline HMGB1 + MLKL & 0.74 & 73.91 \\
\hline HMGB1 + RIPK1 & 0.76 & 74.03 \\
\hline RIPK3 & 0.78 & 91.99 \\
\hline MLKL + RIPK3 & 0.77 & 93.11 \\
\hline MLKL & 0.60 & 105.61 \\
\hline MLKL + RIPK1 & 0.61 & 106.45 \\
\hline RIPK1 & 0.57 & 107.52
\end{tabular}

Classification accuracies (\%) and AICs values of the training data from day E to E-3

Table 3. Classification of the severity status on multiple days on and before the censoring event.

The classification performance of predicting COVID-19 severity using the training and test data consisting of combined RIPK3 and HMGB1 plasma levels on day E to E-3.

$\mathrm{Cl}$, confidence interval; Acc, accuracy

Table 4. Classifier evaluation of the training, test, and validation data. 


\begin{tabular}{|c|c|c|c|c|c|c|c|c|c|c|c|c|c|}
\hline & day & no & n1 & Accuracy & Acc $2.5 \% \mathrm{Cl}$ & \multicolumn{2}{|c|}{ Acc $97.5 \% \mathrm{Cl}$} & \multicolumn{2}{|c|}{ Sensitivity } & Specificity & & & \\
\hline \multirow[t]{4}{*}{ Training data } & $E$ & 12 & 3 & 1.00 & 0.78 & 1.00 & & 1.00 & \multicolumn{2}{|c|}{1.00} & & & \\
\hline & $E-1$ & 10 & 6 & 1.00 & 0.79 & 1.00 & & 1.00 & \multicolumn{2}{|c|}{1.00} & & & \\
\hline & $\mathrm{E}-2$ & 9 & 8 & 0.82 & 0.57 & 0.96 & & 0.89 & \multicolumn{2}{|c|}{0.75} & & & \\
\hline & E-3 & 11 & 5 & 1.00 & 0.79 & 1.00 & & 1.00 & \multicolumn{2}{|c|}{1.00} & & & \\
\hline \multirow[t]{4}{*}{ Test data } & $E$ & 27 & 19 & 0.76 & 0.61 & 0.87 & & 0.93 & \multicolumn{2}{|c|}{0.53} & & & \\
\hline & $\mathrm{E}-1$ & 27 & 19 & 0.72 & 0.57 & 0.84 & & 0.67 & \multicolumn{2}{|c|}{0.79} & & & \\
\hline & $\mathrm{E}-2$ & 27 & 19 & 0.78 & 0.64 & 0.89 & & 0.82 & \multicolumn{2}{|c|}{0.74} & & & \\
\hline & E-3 & 27 & 19 & 0.83 & 0.69 & 0.92 & & 1.00 & \multicolumn{2}{|c|}{0.58} & & & \\
\hline \multicolumn{2}{|l|}{ Training data } & \multicolumn{3}{|c|}{ reference } & \multirow[t]{2}{*}{ Test data } & & \multicolumn{3}{|c|}{ reference } & \multirow{2}{*}{\multicolumn{2}{|c|}{ Validation data }} & \multicolumn{2}{|l|}{ reference } \\
\hline & & & derate & severe & & & mo & lerate & severe & & & moderate & severe \\
\hline \multirow[t]{2}{*}{ predicted } & moderate & 21 & & 0 & predicted & moderate & \multicolumn{2}{|c|}{24} & 5 & \multirow[t]{2}{*}{ predicted } & moderate & 57 & 11 \\
\hline & ere & 0 & & 11 & & severe & 3 & & 14 & & severe & 6 & 35 \\
\hline \multicolumn{4}{|l|}{ Accuracy } & 1.00 & Accuracy & & & & 0.83 & \multicolumn{2}{|l|}{ Accuracy } & & 0.84 \\
\hline \multicolumn{4}{|l|}{ Sensitivity } & 1.00 & Sensitivity & & & & 0.89 & \multicolumn{2}{|l|}{ Sensitivity } & & 0.90 \\
\hline \multicolumn{4}{|l|}{ Specificity } & 1.00 & Specificity & & & & 0.74 & \multicolumn{2}{|l|}{ Specificity } & & 0.76 \\
\hline
\end{tabular}

Confusion matrices of the classification performances on training, test, and validation data with corresponding accuracy, sensitivity, and specificity

Table 5. Logistic regression parameters of fitting the severity state with RIPK3 and HMGB1 plasma levels.

\begin{tabular}{|c|c|c|c|c|c|c|c|c|}
\hline & $\beta$ & SE & $Z$ value & $2.5 \% \mathrm{Cl}$ & $97.5 \% \mathrm{Cl}$ & p-value & significant & RIPK3 and HMGB1 plasma levels of the total \\
\hline (Intercept) & -2.970 & 0.413 & -7.191 & -3.816 & -2.193 & $>0.001$ & 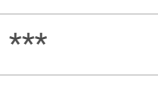 & $\begin{array}{l}\text { measurements are included in a logistic regression to } \\
\text { calculate the odds of disease-related severity change. }\end{array}$ \\
\hline RIPK3 & 0.305 & 0.055 & 5.530 & 0.201 & 0.418 & $>0.001$ & $\star \star \star *$ & The model parameters of the fit are presented \\
\hline HMGB1 & 0.006 & 0.001 & 6.017 & 0.004 & 0.007 & $>0.001$ & 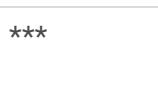 & $\begin{array}{l}(* \star \star p<0.001) \\
\beta, \text { regression coefficient; } S E \text {, standard error }\end{array}$ \\
\hline
\end{tabular}

Table 6. Training set for COVID-19 severity classification using multiple inflammatory variables.

Data is split into severe and moderate as described in our main method section

Acc, accuracy

Table 7. Test set for COVID-19 severity classification using multiple inflammatory variables. 


\begin{tabular}{|c|c|c|c|c|c|c|}
\hline Variables & $\begin{array}{l}\text { Accuracy } \\
\text { (\%) }\end{array}$ & $\begin{array}{l}\mathrm{Acc} 2.5 \% \\
\mathrm{Cl}\end{array}$ & Acc $97.5 \% \mathrm{Cl}$ & p-value & Sensitivity & Specificity \\
\hline HMGB1 + RIPK3 + PCT & 0.931 & 0.862 & 0.972 & 0 & 0.966 & 0.884 \\
\hline HMGB1 + RIPK3 + IL-6 + PCT & 0.931 & 0.862 & 0.972 & 0 & 0.966 & 0.884 \\
\hline HMGB1 + RIPK3 + CRP + IL-6 + PCT & 0.931 & 0.862 & 0.972 & 0 & 0.966 & 0.884 \\
\hline HMGB1 + RIPK3 + PCT + LDH & 0.931 & 0.862 & 0.972 & 0 & 0.966 & 0.884 \\
\hline HMGB1 + RIPK3 + IL-6 + PCT + LDH & 0.931 & 0.862 & 0.972 & 0 & 0.966 & 0.884 \\
\hline HMGB1 + RIPK3 + CRP + IL-6 + PCT + LDH & 0.931 & 0.862 & 0.972 & 0 & 0.966 & 0.884 \\
\hline HMGB1 + RIPK3 + IL-6 + PCT + Leukocyte count & 0.931 & 0.862 & 0.972 & 0 & 0.966 & 0.884 \\
\hline HMGB1 + RIPK3 + CRP + IL-6 + PCT + Leukocyte count & 0.931 & 0.862 & 0.972 & 0 & 0.966 & 0.884 \\
\hline HMGB1 + RIPK3 + IL-6 + PCT + LDH + Leukocyte count & 0.931 & 0.862 & 0.972 & 0 & 0.983 & 0.86 \\
\hline HMGB1 + RIPK3 + CRP + PCT & 0.921 & 0.85 & 0.965 & 0 & 0.948 & 0.884 \\
\hline HMGB1 + RIPK3 + PCT + Leukocyte count & 0.921 & 0.85 & 0.965 & 0 & 0.948 & 0.884 \\
\hline HMGB1 + RIPK3 + CRP + PCT + Leukocyte count & 0.921 & 0.85 & 0.965 & 0 & 0.948 & 0.884 \\
\hline HMGB1 + RIPK3 + PCT + LDH + Leukocyte count & 0.921 & 0.85 & 0.965 & 0 & 0.966 & 0.86 \\
\hline HMGB1 + RIPK3 + CRP + PCT + LDH + Leukocyte count & 0.921 & 0.85 & 0.965 & 0 & 0.966 & 0.86 \\
\hline $\begin{array}{l}\text { HMGB1 + RIPK3 + CRP + IL-6 + PCT + LDH + Leukocyte } \\
\text { count }\end{array}$ & 0.921 & 0.85 & 0.965 & 0 & 0.966 & 0.86 \\
\hline HMGB1 + RIPK3 + CRP + PCT + LDH & 0.911 & 0.838 & 0.958 & 0 & 0.948 & 0.86 \\
\hline HMGB1 + RIPK3 + CRP + IL-6 + LDH & 0.891 & 0.813 & 0.944 & 0 & 0.931 & 0.837 \\
\hline RIPK3 + IL-6 + PCT + Leukocyte count & 0.891 & 0.813 & 0.944 & 0 & 0.966 & 0.791 \\
\hline RIPK3 + CRP + IL-6 + PCT + Leukocyte count & 0.891 & 0.813 & 0.944 & 0 & 0.966 & 0.791 \\
\hline HMGB1 + RIPK3 + CRP + IL-6 + LDH + Leukocyte count & 0.891 & 0.813 & 0.944 & 0 & 0.931 & 0.837 \\
\hline HMGB1 + RIPK3 + CRP + IL-6 & 0.881 & 0.802 & 0.937 & 0 & 0.931 & 0.814 \\
\hline HMGB1 + RIPK3 + CRP + LDH & 0.881 & 0.802 & 0.937 & 0 & 0.931 & 0.814 \\
\hline HMGB1 + RIPK3 + CRP + IL-6 + Leukocyte count & 0.881 & 0.802 & 0.937 & 0 & 0.931 & 0.814 \\
\hline RIPK3 + PCT + Leukocyte count & 0.881 & 0.802 & 0.937 & 0 & 0.966 & 0.767 \\
\hline RIPK3 + CRP + PCT + Leukocyte count & 0.881 & 0.802 & 0.937 & 0 & 0.966 & 0.767 \\
\hline HMGB1 + CRP + IL-6 + PCT + Leukocyte count & 0.881 & 0.802 & 0.937 & 0 & 0.948 & 0.791 \\
\hline HMGB1 + RIPK3 + LDH + Leukocyte count & 0.881 & 0.802 & 0.937 & 0 & 0.931 & 0.814 \\
\hline HMGB1 + RIPK3 + CRP + LDH + Leukocyte count & 0.881 & 0.802 & 0.937 & 0 & 0.931 & 0.814 \\
\hline RIPK3 + IL-6 + PCT + LDH + Leukocyte count & 0.881 & 0.802 & 0.937 & 0 & 0.966 & 0.767 \\
\hline $\mathrm{RIPK} 3+\mathrm{CRP}+\mathrm{PCT}+\mathrm{LDH}$ & 0.871 & 0.79 & 0.93 & 0 & 0.966 & 0.744 \\
\hline HMGB1 + CRP + IL-6 + PCT + LDH & 0.871 & 0.79 & 0.93 & 0 & 0.948 & 0.767 \\
\hline HMGB1 + RIPK3 + CRP + Leukocyte count & 0.871 & 0.79 & 0.93 & 0 & 0.931 & 0.791 \\
\hline HMGB1 + IL-6 + PCT + Leukocyte count & 0.871 & 0.79 & 0.93 & 0 & 0.948 & 0.767 \\
\hline HMGB1 + IL-6 + PCT + LDH + Leukocyte count & 0.871 & 0.79 & 0.93 & 0 & 0.948 & 0.767 \\
\hline HMGB1 + CRP + IL-6 + PCT + LDH + Leukocyte count & 0.871 & 0.79 & 0.93 & 0 & 0.948 & 0.767 \\
\hline RIPK3 + CRP + IL-6 + PCT + LDH + Leukocyte count & 0.871 & 0.79 & 0.93 & 0 & 0.966 & 0.744 \\
\hline HMGB1 + RIPK3 & 0.861 & 0.778 & 0.922 & 0 & 0.897 & 0.814 \\
\hline HMGB1 + RIPK3 + CRP & 0.861 & 0.778 & 0.922 & 0 & 0.914 & 0.791 \\
\hline HMGB1 + RIPK3 + IL-6 & 0.861 & 0.778 & 0.922 & 0 & 0.897 & 0.814 \\
\hline
\end{tabular}




\begin{tabular}{|c|c|c|c|c|c|c|}
\hline Variables & $\begin{array}{l}\text { Accuracy } \\
(\%)\end{array}$ & $\begin{array}{l}\mathrm{Acc} 2.5 \% \\
\mathrm{Cl}\end{array}$ & Acc $97.5 \% \mathrm{Cl}$ & p-value & Sensitivity & Specificity \\
\hline HMGB1 + CRP + LDH & 0.93 & 0.809 & 0.985 & 0 & 1 & 0.812 \\
\hline HMGB1 + CRP + IL-6 + LDH & 0.93 & 0.809 & 0.985 & 0 & 1 & 0.812 \\
\hline HMGB1 + CRP & 0.884 & 0.749 & 0.961 & 0 & 0.963 & 0.75 \\
\hline HMGB1 + IL-6 & 0.884 & 0.749 & 0.961 & 0 & 1 & 0.688 \\
\hline HMGB1 + CRP + IL-6 & 0.884 & 0.749 & 0.961 & 0 & 0.963 & 0.75 \\
\hline HMGB1 + PCT & 0.884 & 0.749 & 0.961 & 0 & 1 & 0.688 \\
\hline HMGB1 + CRP + PCT & 0.884 & 0.749 & 0.961 & 0 & 1 & 0.688 \\
\hline HMGB1 + IL-6 + PCT & 0.884 & 0.749 & 0.961 & 0 & 1 & 0.688 \\
\hline HMGB1 + CRP + IL-6 + PCT & 0.884 & 0.749 & 0.961 & 0 & 1 & 0.688 \\
\hline HMGB1 + IL-6 + LDH & 0.884 & 0.749 & 0.961 & 0 & 1 & 0.688 \\
\hline HMGB1 + PCT + LDH & 0.884 & 0.749 & 0.961 & 0 & 1 & 0.688 \\
\hline HMGB1 + CRP + PCT + LDH & 0.884 & 0.749 & 0.961 & 0 & 1 & 0.688 \\
\hline HMGB1 + IL-6 + PCT + LDH & 0.884 & 0.749 & 0.961 & 0 & 1 & 0.688 \\
\hline HMGB1 + CRP + IL-6 + PCT + LDH & 0.884 & 0.749 & 0.961 & 0 & 1 & 0.688 \\
\hline HMGB1 + CRP + Leukocyte count & 0.884 & 0.749 & 0.961 & 0 & 0.963 & 0.75 \\
\hline HMGB1 + IL-6 + Leukocyte count & 0.884 & 0.749 & 0.961 & 0 & 1 & 0.688 \\
\hline HMGB1 + CRP + IL-6 + Leukocyte count & 0.884 & 0.749 & 0.961 & 0 & 0.963 & 0.75 \\
\hline HMGB1 + CRP + PCT + Leukocyte count & 0.884 & 0.749 & 0.961 & 0 & 1 & 0.688 \\
\hline HMGB1 + CRP + IL-6 + PCT + Leukocyte count & 0.884 & 0.749 & 0.961 & 0 & 1 & 0.688 \\
\hline HMGB1 + CRP + LDH + Leukocyte count & 0.884 & 0.749 & 0.961 & 0 & 0.963 & 0.75 \\
\hline HMGB1 + IL-6 + LDH + Leukocyte count & 0.884 & 0.749 & 0.961 & 0 & 1 & 0.688 \\
\hline RIPK3 + IL-6 + LDH + Leukocyte count & 0.884 & 0.749 & 0.961 & 0 & 0.926 & 0.812 \\
\hline HMGB1 + CRP + IL-6 + LDH + Leukocyte count & 0.884 & 0.749 & 0.961 & 0 & 0.963 & 0.75 \\
\hline HMGB1 + CRP + PCT + LDH + Leukocyte count & 0.884 & 0.749 & 0.961 & 0 & 1 & 0.688 \\
\hline HMGB1 + CRP + IL-6 + PCT + LDH + Leukocyte count & 0.884 & 0.749 & 0.961 & 0 & 1 & 0.688 \\
\hline HMGB1 + RIPK3 + CRP & 0.86 & 0.721 & 0.947 & 0.001 & 0.889 & 0.812 \\
\hline HMGB1 + RIPK3 + CRP + IL-6 & 0.86 & 0.721 & 0.947 & 0.001 & 0.926 & 0.75 \\
\hline RIPK3 + PCT & 0.86 & 0.721 & 0.947 & 0.001 & 0.926 & 0.75 \\
\hline RIPK3 + CRP + PCT & 0.86 & 0.721 & 0.947 & 0.001 & 0.926 & 0.75 \\
\hline $\mathrm{HMGB} 1+\mathrm{RIPK} 3$ + CRP + LDH & 0.86 & 0.721 & 0.947 & 0.001 & 0.889 & 0.812 \\
\hline RIPK3 + PCT + LDH & 0.86 & 0.721 & 0.947 & 0.001 & 0.926 & 0.75 \\
\hline RIPK3 + CRP + PCT + LDH & 0.86 & 0.721 & 0.947 & 0.001 & 0.926 & 0.75 \\
\hline HMGB1 + RIPK3 + Leukocyte count & 0.86 & 0.721 & 0.947 & 0.001 & 0.926 & 0.75 \\
\hline RIPK3 + CRP + Leukocyte count & 0.86 & 0.721 & 0.947 & 0.001 & 0.889 & 0.812 \\
\hline HMGB1 + RIPK3 + CRP + Leukocyte count & 0.86 & 0.721 & 0.947 & 0.001 & 0.889 & 0.812 \\
\hline RIPK3 + CRP + IL-6 + Leukocyte count & 0.86 & 0.721 & 0.947 & 0.001 & 0.889 & 0.812 \\
\hline HMGB1 + PCT + Leukocyte count & 0.86 & 0.721 & 0.947 & 0.001 & 1 & 0.625 \\
\hline RIPK3 + CRP + PCT + Leukocyte count & 0.86 & 0.721 & 0.947 & 0.001 & 0.926 & 0.75 \\
\hline HMGB1 + RIPK3 + LDH + Leukocyte count & 0.86 & 0.721 & 0.947 & 0.001 & 0.926 & 0.75 \\
\hline RIPK3 + CRP + LDH + Leukocyte count & 0.86 & 0.721 & 0.947 & 0.001 & 0.889 & 0.812 \\
\hline
\end{tabular}




\begin{tabular}{|c|c|c|c|c|c|c|}
\hline HMGB1 + RIPK3 + CRP + LDH + Leukocyte count & 0.86 & 0.721 & 0.947 & 0.001 & 0.889 & 0.812 \\
\hline HMGB1 + RIPK3 + IL-6 + LDH + Leukocyte count & 0.86 & 0.721 & 0.947 & 0.001 & 0.926 & 0.75 \\
\hline RIPK3 + CRP + IL-6 + LDH + Leukocyte count & 0.86 & 0.721 & 0.947 & 0.001 & 0.889 & 0.812 \\
\hline HMGB1 + PCT + LDH + Leukocyte count & 0.86 & 0.721 & 0.947 & 0.001 & 1 & 0.625 \\
\hline RIPK3 + PCT + LDH + Leukocyte count & 0.86 & 0.721 & 0.947 & 0.001 & 0.926 & 0.75 \\
\hline HMGB1 + RIPK3 + PCT + LDH + Leukocyte count & 0.86 & 0.721 & 0.947 & 0.001 & 0.926 & 0.75 \\
\hline RIPK3 + CRP + PCT + LDH + Leukocyte count & 0.86 & 0.721 & 0.947 & 0.001 & 0.926 & 0.75 \\
\hline HMGB1 + RIPK3 + CRP + PCT + LDH + Leukocyte count & 0.86 & 0.721 & 0.947 & 0.001 & 0.926 & 0.75 \\
\hline HMGB1 + IL-6 + PCT + LDH + Leukocyte count & 0.86 & 0.721 & 0.947 & 0.001 & 0.963 & 0.688 \\
\hline RIPK3 + IL-6 + PCT + LDH + Leukocyte count & 0.86 & 0.721 & 0.947 & 0.001 & 0.926 & 0.75 \\
\hline HMGB1 + RIPK3 + IL-6 + PCT + LDH + Leukocyte count & 0.86 & 0.721 & 0.947 & 0.001 & 0.926 & 0.75 \\
\hline RIPK3 + CRP + IL-6 + PCT + LDH + Leukocyte count & 0.86 & 0.721 & 0.947 & 0.001 & 0.926 & 0.75 \\
\hline $\begin{array}{l}\text { HMGB1 + RIPK3 + CRP + IL-6 + PCT + LDH + Leukocyte } \\
\text { count }\end{array}$ & 0.86 & 0.721 & 0.947 & 0.001 & 0.926 & 0.75 \\
\hline HMGB1 + RIPK3 & 0.837 & 0.693 & 0.932 & 0.002 & 0.926 & 0.688 \\
\hline RIPK3 + CRP & 0.837 & 0.693 & 0.932 & 0.002 & 0.889 & 0.75 \\
\hline HMGB1 + RIPK3 + IL-6 & 0.837 & 0.693 & 0.932 & 0.002 & 0.926 & 0.688 \\
\hline HMGB1 + RIPK3 + PCT & 0.837 & 0.693 & 0.932 & 0.002 & 0.926 & 0.688 \\
\hline
\end{tabular}

Data is split into severe and moderate

Acc, accuracy

\section{Figures}

Fig. 1
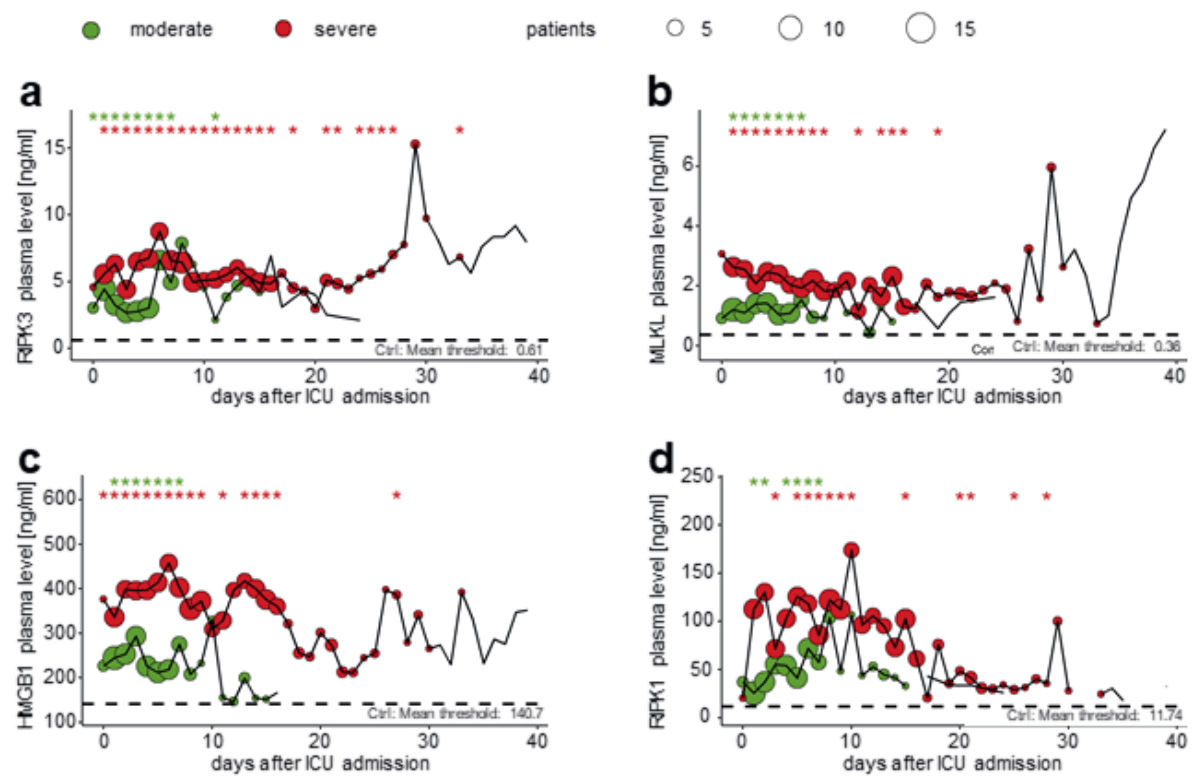

Figure 1 
Elevated RIPK3, MLKL, HMGB1, and RIPK1 plasma levels in COVID 19 intensive care patients. Mean longitudinal levels of the COVID 19 cohort with 28 patients with moderate (green) and 18 patients with severe (red) COVID 19 are presented. Bubble size is equivalent to the number of patients. (a) RIPK3, (b) MLKL, (c) HMGB1, and (d) RIPK1 plasma levels are plotted by days after ICU admission. Significant differences between patients with moderate COVID 19 and healthy controls (Ctrl) were found on days 0 to 7 and 11 (RIPK3); 1 to 7 (MLKL and HMGB1); 1, 2, and 4 to 7 (RIPK1). Significant differences between patients with severe COVID 19 and healthy controls were found on days 1 to $16,18,21,22,24$ to 27 , and 33 (RIPK3); 1 to $9,12,14$ to 16 , and 19 (MLKL); 0 to 9, 11, 13 to 16, and 27 (HMGB1); 3, 5 to 10, 15, 20, 21, 25, and 28 (RIPK1). Statistical differences between plasma levels of patients with moderate and severe COVID 19 and 15 healthy controls (black dotted line) were assessed using unpaired two sided

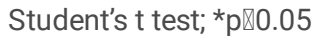

\section{Fig. 2}

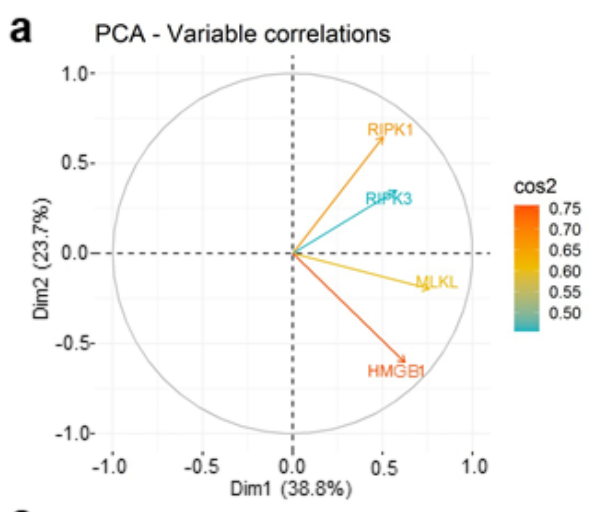

C PCA - Contributions (Dimension 1)

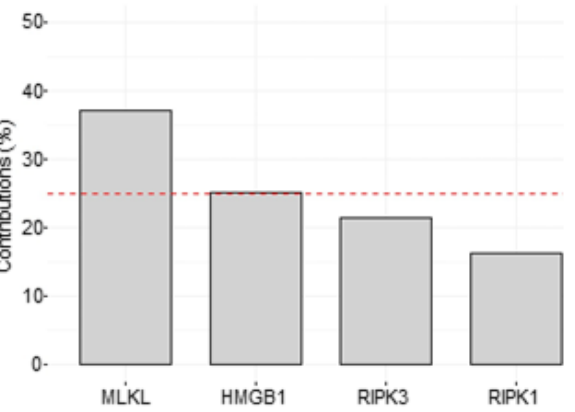

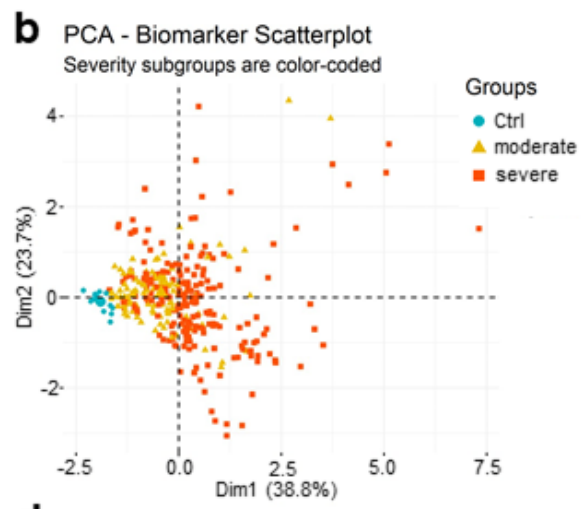

d PCA - Contributions (Dimension 2)

50 .

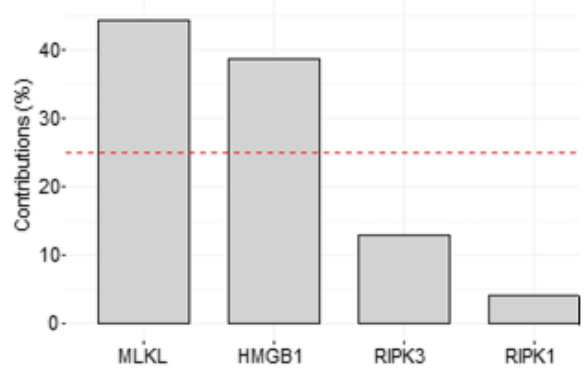

Figure 2

PCA of RIPK3, MLKL, HMGB1, and RIPK1 plasma levels in COVID 19 intensive care patients. (a) PCA variable correlations plot of RIPK3, MLKL, HMGB1, and RIPK1 plasma levels. With two dimensions, $62.5 \%$ of the variance is expressed. (b) PCA scatter plot of the three groups (healthy control=Ctrl, blue circle; moderate COVID 19=yellow triangle; severe COVID 19=orange square) with plasma levels of all four markers. (c) Bar plot of variables' (RIPK3, MLKL, HMGB1, and RIPK1) contribution to Dim1 in percentage. The red dashed line indicates the expected average contribution (25\%). (d) Bar plot of the same variables' contribution to Dim2 in percentage. Dim, dimension; cos2, squared coordinates (quality of representation) 
Fig. 3
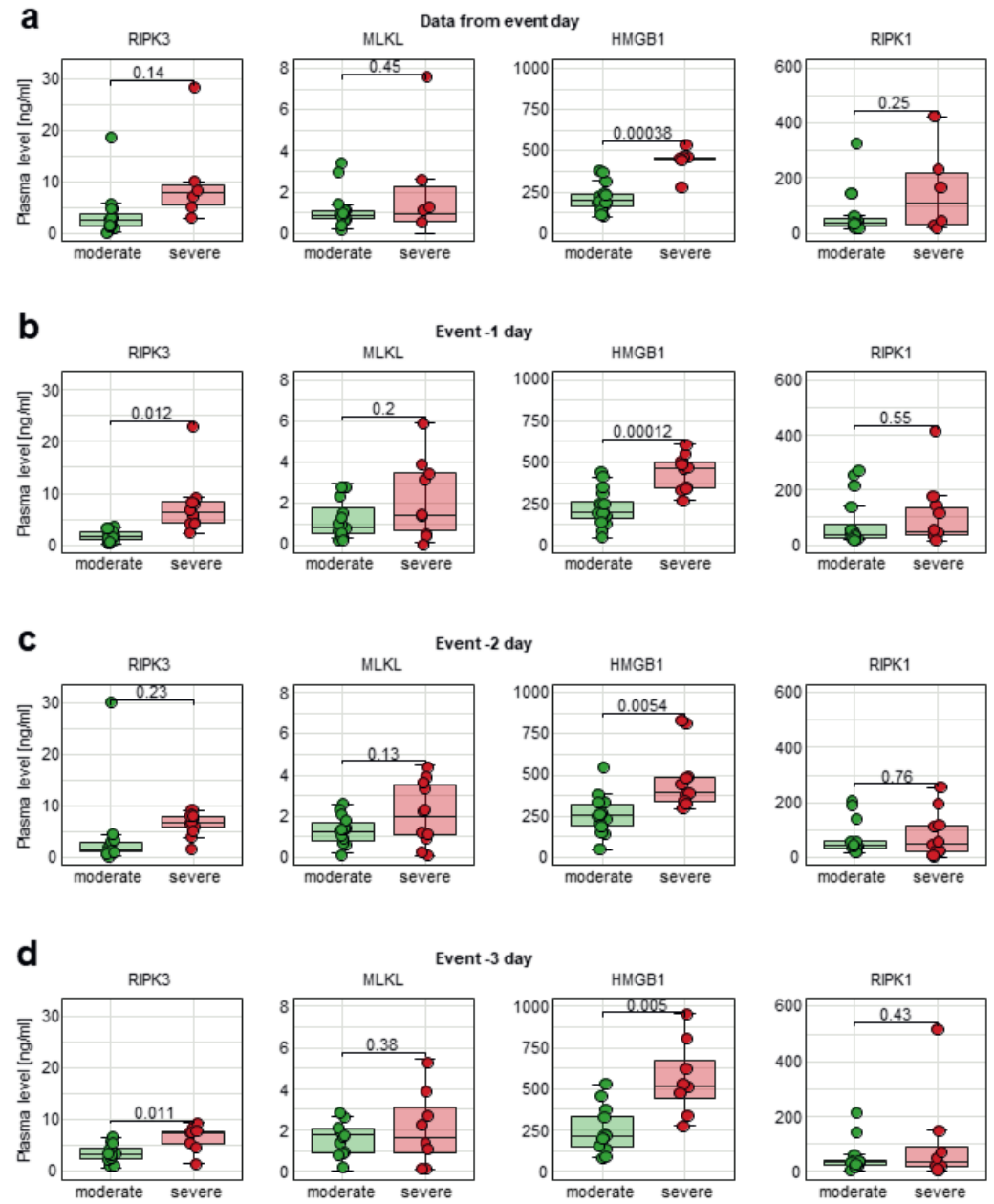

\section{Figure 3}

Elevated RIPK3 and HMGB1 plasma levels in patients with severe COVID 19 progression. Box plots of RIPK3, MLKL, HMGB1, and RIPK1 plasma levels on (a) the last day on the ICU=E including 15 patients with moderate and 6 patients with severe COVID 19, (b) one day before=E 1 including 16 patients with moderate and 10 patients with severe COVID 19, (c) two days before=E 2 including 14 patients with moderate and 11 patients with severe COVID 19, and (d) three days before=E 3 including 13 patients with moderate and 8 patients with severe COVID 19. Statistical differences between patients with moderate and severe COVID 19 were assessed using an unpaired two sided Student's t test and were regarded significant at $\mathrm{p} \otimes 0.05$ 
Fig. 4

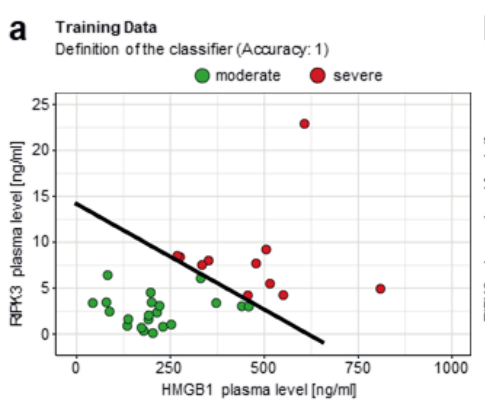

b Test Data

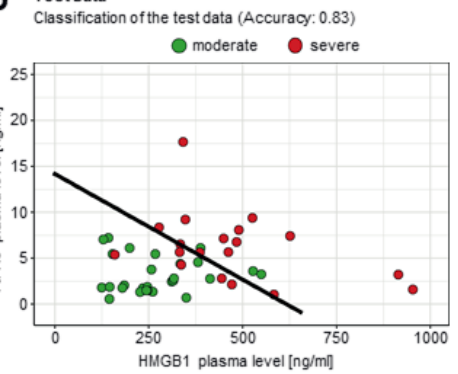

C Validation Data Validation of the classifier (Acauracy. 0.84) - moderate severe

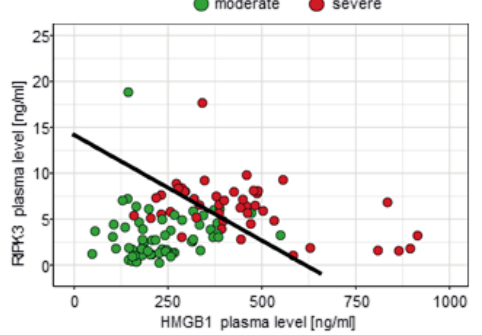

d Accuracy decay overtime

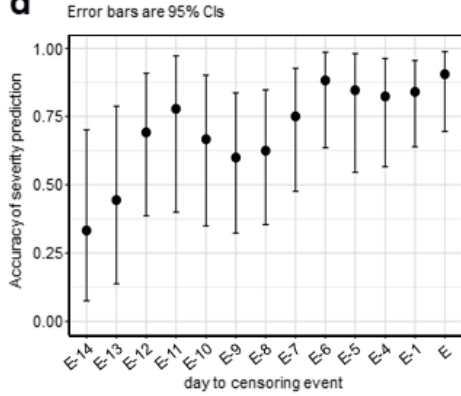

e RIPK3

Error bars are $95 \% \mathrm{Cls}$

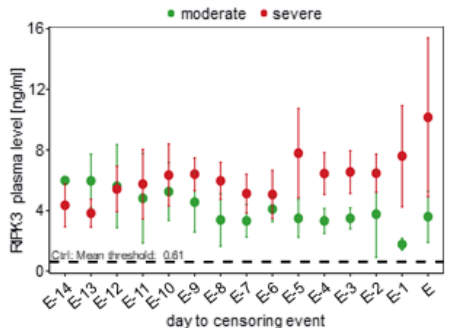

f HMGB1

Error bars are $95 \%$ Cls

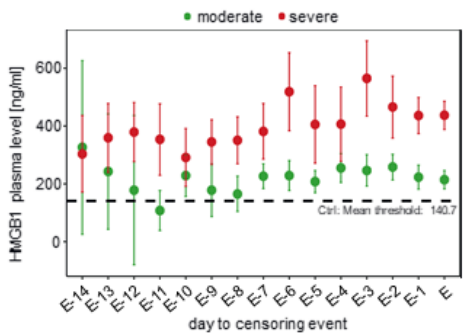

day to censoring event

\section{Figure 4}

Classification evaluation. From the coefficients of the generalized linear model (GLM), a linear discriminator can be constructed and projected into the feature space (black line), constituted by the measured plasma levels of RIPK3 and HMGB1 (moderate=green, severe=red). (a) The training data from the days E 1 and E 3 that were used in building the classifier are shown in a scatterplot, separated by the linear classifier. (b) Projection of the test data into feature space, using the discriminator to evaluate the classification performance. Five red dots are classified as green (moderate) and three green dots are classified as red (severe). Some dots are close to the discriminator with high classification ambiguity. (c) Validation of the classifier using data from up to day E 7 (excluding E 1 and E 3). The fraction of false negatives is larger (11 dots) than the false positives ( 6 dots). (d) The classifier's mean prediction accuracy was plotted with $95 \%$ confidence intervals up to 14 days before the event (excluding $E 2$ and $E 3$ ). Mean RIPK3 (e) and HMGB1 (f) plasma levels of patients with moderate (green) and severe (red) COVID 19 are plotted from day E 14 until the censoring event with $95 \%$ confidence intervals. Mean plasma levels of healthy controls (Ctrl) are represented as black dashed lines

\section{Supplementary Files}

This is a list of supplementary files associated with this preprint. Click to download.

- STROBEchecklistv4combined.docx

- SupplementNOTrackChanges.docx 